\title{
Interference Exploitation based Secure Transmission for Distributed Antenna Systems
}

\author{
$1^{\text {st }}$ Zhongxiang Wei, $2^{\text {nd }}$ Christos Masouros, and $3^{\text {rd }}$ Fan Liu \\ Department of Electronic and Electrical Engineering \\ University College London \\ London, UK \\ \{zhongxiang.wei, c.masouros, fan.liu\}@ucl.ac.uk
}

\begin{abstract}
Distributed antenna (DA) is considered as a strong alternative to conventional centralized multiple-input multipleoutput (MIMO), to provide a greener and user-centric network structure. However, physical layer (PHY) security becomes more challenging in DA systems because of the proximity to the transmitters. In this paper, we jointly optimize DA activation/deactivation and secure precoding to minimize the total power consumption, subjected to legitimate user's (LU) qualityof-service (QoS) and PHY security constraints against potential eavesdroppers (Eves). A practical scenario is considered, where channel state information (CSI) of all the nodes can only be imperfectly obtained. In the presence of infinite probabilities of CSI uncertainties, a deterministic robust based algorithm is designed to always satisfy LU's QoS requirement and address PHY security constraints against Eves. Moreover, essentially different from existing artificial noise (AN)-aided secure transmission schemes, where $A N$ ' leakage effect at $L U$ needs to be suppressed, we utilize $A N$ as a beneficial element at $L U$ end while keeping it destructive at potential Eves. Simulation results verify that, the proposed algorithm incurs much lower power consumption compared to its benchmarks, thanks to the additional degrees of freedom of antenna selection and utilizing constructive AN. Last but not least, by adaptively facilitating DA activation/deactivation, the proposed algorithm addresses a user-centric network structure, which is more flexible over the conventional centralized MIMO systems.

Index Terms-User centric systems, Physical layer security, Constructive artificial noise, Secure Precoding, Antenna selection
\end{abstract}

\section{INTRODUCTION}

With the increasing interest in physical layer (PHY) security, different techniques and signal processing approaches have been extensively investigated [1]. Among them, centralized antenna (CA) multiple-input multiple-output (MIMO) has been considered as a potential technique for enhancing PHY security thanks to its high spatial diversity [2] [3]. However, it is worth noting that, centralized MIMO requires high power consumption caused by its fully activated radio-frequency (RF) chains, which is against the green evolution proposed by the next generation communication systems [4] [5]. Besides, since all the antennas are co-located together, centralized MIMO often suffers from an equal level of path loss (PL) from the antenna array to one user, and edge users may not be well served due to the severe propagation attenuation [6]. To alleviate the aforementioned problems, distributed antenna (DA) systems have been proposed to achieve a green, user-centric and flexible network structure. Explicitly, the geographically positioned antennas help reduce the communication distance, and hence transmission power can be eased. Since the power consumption of an active DA node mainly comes from digital/analog converter, analog/digital converter, filter, synthesizer, mixers, etc [8], the power consumption of a DA node can be significantly reduced by switching it off [3]. By adaptively activating those DAs contributing the most, DA systems can provide on-demand service for users [7] [8].

Nevertheless, it should be pointed that due to the proximity to transmitters, it is also easier for potential eavesdroppers (Eves) to grasp the confidential messages intended for the legitimate user (LU). Hence, PHY security in DA systems becomes more challenging, and needs to be carefully addressed. In the past few years, artificial noise (AN)-aided secure transmission has been widely studied to jam Eves. In [9], AN was generated into the orthogonal space of the LU's channel, and thus the potential Eves can be interfered by AN while its leakage is zeroed at the LU. On the other hand, the authors in [10] designed a scheme that AN can be spatially transmitted into the direction of the Eves, on the basis of knowing the Eves' channel state information (CSI). On the specific topic of secure transmission in DA systems, the authors in [11] maximized the ergodic secrecy rate in DA systems, where AN and confidential signal are jointly designed and transmitted at each DA. In [3], the power minimization problem was demonstrated for DA systems, where the potential Eves are considered as idle LUs and scavenge energy from AN. The authors in [12] maximized the LU's signal-to-interference-plus-noise ratio (SINR) requirement, subjected to PHY security constraint.

The aforementioned research, however, treated AN as a harmful element and suppressed its leakage at the LU as much as possible. If AN can be beneficial to the $\mathrm{LU}$, based on the concept of constructive interference (CI), it may help improve the LU's receiving performance. CI was firstly introduced in code division multiple access system [13] [14] [15], and subsequent research applied this technique into MIMO [16], cognitive radio [17] [18] and constant envelop systems [19] [20]. Based on the aforementioned work, the authors in [21] proposed a scheme to utilize AN in a CA multiple-input singleoutput (MISO) system, where AN at the transmitter was aligned with the received signal at the LU. However, enormous power consumption is incurred by the fully activated antennas [21]. 
Motivated by the aforementioned issues, we propose a joint DA selection and secure precoding design to minimize total power consumption for DA systems, under infinite probabilities of CSI uncertainties. Our contributions are summarized in the following:

- DA activation/deactivation and secure precoding are jointly designed to fully utilize the additional degrees of freedom in antenna selection and beneficial effect of constructive AN, which significantly reduces the total power consumption yet addressing the LU's SINR requirement and PHY security constraints against the Eves.

- A robust power minimization oriented optimization problem is formulated, where CSI can only be imperfectly obtained. In the presence of CSI error, a novel algorithm is proposed to solve the optimization problem in terms of deterministic robust optimization, where the LU's SINR and PHY security against the Eves are always guaranteed with all the possible CSI uncertainties.

- A power efficient and user-centric network structure is demonstrated by the proposed algorithm. Explicitly, the working status of each DA is flexibly determined by the LU and Eves' positions to provide on-demand services: the DAs close to users have higher probabilities of activation, while the DAs far from users have higher probabilities of being sleeping for saving power.

Notations: Matrices and vectors are represented by boldface capital and lower case letters, respectively. $|\cdot|$ denotes the absolute value of a complex scalar. $\boldsymbol{A}^{H} \boldsymbol{A}^{T}, \operatorname{Tr}(\boldsymbol{A}), \operatorname{Rank}(\boldsymbol{A})$ denotes the Hermitian, transpose and trace of matrix $\boldsymbol{A}$. Operator diag (.) stacks the elements into a diagonal matrix. $\boldsymbol{A} \succeq 0$ means $\boldsymbol{A}$ is a positive semi-definite matrix. $\|\cdot\|_{p}$ means the p-norm of a vector or a matrix. $\boldsymbol{I}_{n}$ means a $n$-by- $n$ identity matrix. Subscripts R and I represent the real part and imaginary parts, respectively. $\mathbb{C}^{N \times M}$ and $\mathbb{H}^{N \times M}$ denote sets of all $N \times M$ matrices and Hermitian matrices with complex entries. $\mathcal{C N}(a, b)$ denotes a normal distributed variable with expectation $a$ and variance $b$.

\section{System Model And Constructive Interference}

In this section, system model is introduced in II-A and the concept of CI is briefly discussed in II-B.

\section{A. System Model}

We consider a DA system at downlink. All $N$ DA are connected to a signal processing center through a noise-free wired front-haul, where confidential messages are sent to the $\mathrm{LU}$ in the presence of $K$ potential Eves. The LU and Eves are all equipped with single antenna for simplicity. CSI is obtained by channel estimation in the training phase as in [22], and the channels of all the nodes are given as

$$
\boldsymbol{h}_{u}=\hat{\boldsymbol{h}_{u}}+\boldsymbol{e}_{u} \text { and } \boldsymbol{h}_{k}=\hat{\boldsymbol{h}_{k}}+\boldsymbol{e}_{k}, \forall k \in K,
$$

where the estimated channel between the DAs and the LU is represented by $\hat{\boldsymbol{h}_{u}} \in \mathbb{C}^{N \times 1}$ with estimation error $\boldsymbol{e}_{u}$. $\hat{\boldsymbol{h}_{k}} \in$ $\mathbb{C}^{N \times 1}$ denotes the estimated channel between the DAs and the $k$-th Eve with estimation error $\boldsymbol{e}_{k}$. The element of error $\boldsymbol{e}_{u}$ follows $\mathcal{C N}\left\{0, \sigma_{u}^{2}\right\}$ while the element of error vector $\boldsymbol{e}_{k}$ follows $\mathcal{C N}\left\{0, \sigma_{k}^{2}\right\}[3]$.

\section{B. Constructive Interference}

$\mathrm{CI}$ is the interference pushes the received signals away from the detection threshold [13]. Denote $\boldsymbol{w} \in \mathbb{C}^{N \times 1}$ and $\boldsymbol{a} \in \mathbb{C}^{N \times 1}$ as precoding and AN, respectively. Denote $x_{u}=u e^{j \phi_{u}}$ as the confidential symbol for the LU. The received signal at the LU and the $k$-th Eve can be calculated as

$$
y_{u}=\boldsymbol{h}_{u}^{T}\left(\boldsymbol{w} x_{u}+\boldsymbol{a}\right)+n_{u}, y_{k}=\boldsymbol{h}_{k}^{T}\left(\boldsymbol{w} x_{u}+\boldsymbol{a}\right)+n_{k},
$$

where $n_{u} \sim \mathcal{C N}\left(0, \sigma_{n}^{2}\right)$ and $n_{k} \sim \mathcal{C N}\left(0, \sigma_{n}^{2}\right)$ denote the Additive white Gaussian noises (AWGN) at the LU and the $k$-th Eve, respectively. Accordingly, the received SINR at the LU and $k$-th Eve are conventionally denoted as

$$
\Gamma_{u}=\frac{\left|\boldsymbol{h}_{u}^{T} \boldsymbol{w}\right|^{2}}{\sigma_{n}^{2}+\left|\boldsymbol{h}_{u}^{T} \boldsymbol{a}\right|^{2}}, \quad \Gamma_{k}=\frac{\left|\boldsymbol{h}_{k}^{T} \boldsymbol{w}\right|^{2}}{\sigma_{n}^{2}+\left|\boldsymbol{h}_{k}^{T} \boldsymbol{a}\right|^{2}},
$$

where $\Gamma_{u}$ and $\Gamma_{k}$ denote the LU and the $k$-th Eve's SINR, respectively. (3) indicates that AN is treated as an undesired element at the LU, and hence its leakage needs to be minimized at the LU. By contrast, the principle of constructive AN is to align it with the desired signal at the LU. Since the transmitted signal can be also written as $\left(\boldsymbol{w}+\boldsymbol{a} e^{-j \phi_{u}}\right) x_{u}$, according to the geometric interpretation in Fig. 1, the requirement of generating constructive AN can be given as

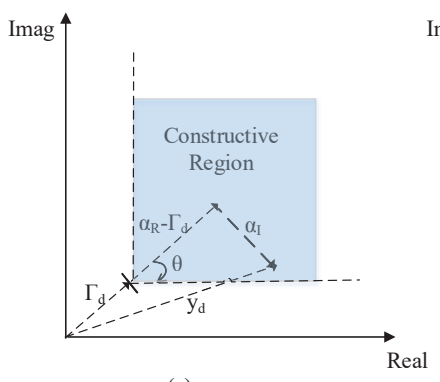

(a)

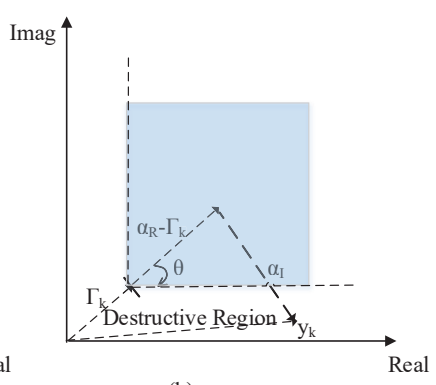

(b)
Fig. 1. Q-PSK modulation is utilized as an example. (a) Constructive artificial noise for LU, where $\alpha_{R}=\Re\left\{\boldsymbol{h}_{u}^{T} \boldsymbol{w}+\boldsymbol{h}_{u}^{T} \boldsymbol{a} e^{-j \phi_{u}}\right\}$ and $\alpha_{I}=\Im\left\{\boldsymbol{h}_{u}^{T} \boldsymbol{w}+\right.$ $\left.\boldsymbol{h}_{u}^{T} \boldsymbol{a} e^{-j \phi_{u}}\right\}$. (b) Destructive artificial noise for potential Eves, where $\alpha_{R}=$ $\Re\left\{\boldsymbol{h}_{k}^{T} \boldsymbol{w}+\boldsymbol{h}_{k}^{T} \boldsymbol{a} e^{-j \phi_{u}}\right\}$ and $\alpha_{I}=\Im\left\{\boldsymbol{h}_{k}^{T} \boldsymbol{w}+\boldsymbol{h}_{k}^{T} \boldsymbol{a} e^{-j \phi_{u}}\right\}$.

$$
\begin{aligned}
& \left|\Im\left\{\boldsymbol{h}_{u}^{T} \boldsymbol{w}+\boldsymbol{h}_{u}^{T} \boldsymbol{a} e^{-j \phi_{u}}\right\}\right| \\
& \quad \leq\left(\Re\left\{\boldsymbol{h}_{u}^{T} \boldsymbol{w}+\boldsymbol{h}_{u}^{T} \boldsymbol{a} e^{-j \phi_{u}}\right\}-\sigma \sqrt{\Gamma_{u}}\right) \tan \theta,
\end{aligned}
$$

where $\theta=\pi / M$ and $M$ is constellation size [21]. As seen from (4), AN is designed to be beneficial for the $\mathrm{LU}$, and the LU' SINR $\Gamma_{u}$ is also embedded.

Accordingly, the received SINR of the LU becomes into

$$
\gamma_{u}=\frac{\left|\boldsymbol{h}_{u}^{T}\left(\boldsymbol{w}+\boldsymbol{a} e^{-j \phi_{u}}\right)\right|^{2}}{\sigma_{n}^{2}},
$$

which indicates that the LU's SINR is improved by utilizing AN. 


\section{Power Minimization with CSI UnCERTAinties}

In section, we first formulate the optimization problem and then present the solution.

\section{A. Problem Formulation}

Define $\boldsymbol{\Delta}$ as the channel estimation uncertainties set, which contains all the possible CSI uncertainties and specifies an ellipsoidal uncertainty region for the estimated CSI [23]. Define $w_{n}$ as the $n$-th element of the precoding weight $\boldsymbol{w} \in \mathbb{C}^{N \times 1}$. Define $a_{n}$ as the $n$-th AN element of the vector $\boldsymbol{a} \in \mathbb{C}^{N \times 1}$. Define $\boldsymbol{t}$ as DA selection vector, whose element $t_{n}=\{0,1\}$ indicates the $n$-th DA is activated or deactivated, respectively. To minimize the total power consumption, we jointly optimize vectors $\boldsymbol{w}, \boldsymbol{a}$ and $\boldsymbol{t}$. Accordingly, the problem is given as

$$
\begin{aligned}
P 1 & \underset{\boldsymbol{w}, \boldsymbol{a}, \boldsymbol{t}}{\operatorname{argmin}} \frac{\| \boldsymbol{w}+\left.\boldsymbol{a} e^{-j \phi_{u}}\right|^{2}}{\eta}+\sum_{n=1}^{N}\left(t_{n} p_{\text {on }}+\left(1-t_{n}\right) p_{o f f}\right), \\
& (C 1): 0 \leq\left|w_{n}+a_{n} e^{-j \phi_{u}}\right|^{2}, \forall n \in N, \\
& (C 2):\left|w_{n}+a_{n} e^{-j \phi_{u}}\right|^{2} \leq t_{n} p_{D A}, \forall n \in N, \\
& (C 3): \min _{\boldsymbol{e}_{u} \in \Delta} \Gamma_{u} \geq \overline{\Gamma_{u}} \\
& (C 4): \max _{\boldsymbol{e}_{\boldsymbol{k}} \in \Delta} \Gamma_{k} \leq \overline{\Gamma_{k}}, k \in K \\
& (C 5): t_{n}=\{0,1\}, \forall n \in N,
\end{aligned}
$$

where $\eta$ denotes the drain efficiency at the DAs. Constraints (C1) and (C2) denote the transmission power at each DA should be non-negative and upper bounded by the maximum available power $p_{D A}$. Constraints $(\mathrm{C} 3)$ and $(\mathrm{C} 4)$ indicate deterministic SINR requirement for the LU and PHY security constraints against the Eves. Explicitly, the LU's worst-case SINR should be no smaller than the threshold $\overline{\Gamma_{u}}$, while the Eves' best-case SINR should be lower than the PHY security threshold $\overline{\Gamma_{k}}$, with infinite probabilities of CSI uncertainties.

1) Optimization Solution: To solve optimization problem P1, we first need to hand the constraints (C3) and (C4) that contain infinite possibilities. Besides, AN should be constructive to the LU while kept destructive to the Eves. According to geometric interpretation in (4), constraints (C3) and (C4) are equivalent to

$$
\begin{aligned}
(C 3): \min _{\boldsymbol{e}_{\boldsymbol{u}} \in \boldsymbol{\Delta}}:\left|\Im\left\{\boldsymbol{h}_{u}^{T} \boldsymbol{w}+\boldsymbol{h}_{u}^{T} \boldsymbol{a} e^{-j \phi_{u}}\right\}\right| \\
\leq\left(\Re\left\{\boldsymbol{h}_{u}^{T} \boldsymbol{w}+\boldsymbol{h}_{u}^{T} \boldsymbol{a} e^{-j \phi_{u}}\right\}-\sigma \sqrt{\overline{\Gamma_{u}}}\right) \tan \theta, \\
(C 4): \max _{\boldsymbol{e}_{\boldsymbol{k}} \in \boldsymbol{\Delta}}:\left|\Im\left\{\boldsymbol{h}_{k}^{T} \boldsymbol{w}+\boldsymbol{h}_{k}^{T} \boldsymbol{z} e^{-j \phi_{u}}\right\}\right| \\
\geq\left(\Re\left\{\boldsymbol{h}_{k}^{T} \boldsymbol{w}+\boldsymbol{h}_{k}^{T} \boldsymbol{a} e^{-j \phi_{u}}\right\}-\sigma \sqrt{\overline{\Gamma_{k}}}\right) \tan \theta,
\end{aligned}
$$

We now first handle constraint (C3). Substituting $\boldsymbol{h}_{u}=\hat{\boldsymbol{h}_{u}}+$ $\boldsymbol{e}_{u}$ into (7), (C3) can be equivalently written as

$$
\begin{aligned}
& (C 3) \min _{\boldsymbol{e}_{u} \in \boldsymbol{\Delta}}:\left|\Im\left\{\left(\hat{\boldsymbol{h}_{u}}+\boldsymbol{e}_{u}\right)^{T} \boldsymbol{w}+\left(\hat{\boldsymbol{h}_{u}}+\boldsymbol{e}_{u}\right)^{T} \boldsymbol{a} e^{-j \phi_{u}}\right\}\right| \leq \\
& \left(\Re\left\{\left(\hat{\boldsymbol{h}_{u}}+\boldsymbol{e}_{u}\right)^{T} \boldsymbol{w}+\left(\hat{\boldsymbol{h}_{u}}+\boldsymbol{e}_{u}\right)^{T} \boldsymbol{a} e^{-j \phi_{u}}\right\}-\sigma \sqrt{\overline{\Gamma_{u}}}\right) \tan \theta,
\end{aligned}
$$

Decomposing the real and imaginary parts, constraint $(C 3)$ is equivalent to the following two inequalities

$$
\left\{\begin{array}{l}
\min _{\boldsymbol{e}_{u} \in \Delta} \sigma_{n} \sqrt{\overline{\Gamma_{u}}} \tan \theta+\hat{\boldsymbol{h}_{I, u}} \boldsymbol{s}_{R}+\hat{\boldsymbol{h}_{R, d}} \boldsymbol{s}_{I}+\boldsymbol{e}_{I, u} \boldsymbol{s}_{R}+\boldsymbol{e}_{R, u} \boldsymbol{s}_{I} \\
-\left(\boldsymbol{h}_{R, u} \boldsymbol{s}_{R}-\boldsymbol{h}_{I, u} \boldsymbol{s}_{I}+\boldsymbol{e}_{R, d} \boldsymbol{s}_{R}-\boldsymbol{e}_{I, u} \boldsymbol{s}_{I}\right) \tan \theta \leq 0, \\
\min _{\boldsymbol{e}_{u} \in \sigma_{n}} \sqrt{\overline{\Gamma_{u}}} \tan \theta-\boldsymbol{h}_{I, u} \boldsymbol{u}_{R}-\boldsymbol{h}_{R, u} \boldsymbol{u}_{I}-\boldsymbol{e}_{R, u} \boldsymbol{s}_{I}-\boldsymbol{e}_{I, u} \boldsymbol{s}_{R} \\
-\left(\boldsymbol{h}_{R, u} \boldsymbol{s}_{R}-\boldsymbol{h}_{I, u} \boldsymbol{s}_{I}+\boldsymbol{e}_{R, u} \boldsymbol{s}_{R}-\boldsymbol{e}_{I, u} \boldsymbol{s}_{I}\right) \tan \theta \leq 0,
\end{array}\right.
$$

where $\boldsymbol{w}+\boldsymbol{a} e^{-j \phi_{u}}=\boldsymbol{s}$.

The first inequality of (9) can be rewritten as

$$
\min _{\boldsymbol{e}_{u} \in \Delta}\left[\boldsymbol{e}_{I, u}-\boldsymbol{e}_{R, u} \tan \theta ; \boldsymbol{e}_{R, u}+\boldsymbol{e}_{I, u} \tan \theta\right]^{T}\left[\boldsymbol{s}_{R} ; \boldsymbol{s}_{I}\right]+\varrho_{u, 1} \leq 0
$$

where $\varrho_{u, 1}=\sigma_{n} \sqrt{\overline{\Gamma_{u}}} \tan \theta+\hat{\boldsymbol{h}_{I, u}} \boldsymbol{s}_{R}+\hat{\boldsymbol{h}_{R, u}} \boldsymbol{s}_{I}-\hat{\boldsymbol{h}_{R, u}} \boldsymbol{s}_{R} \tan \theta+$ $\hat{\boldsymbol{h}_{I, u}} \boldsymbol{s}_{I} \tan \theta$. To handle the infinite CSI uncertainties in (10), we transform (10) into a linear matrix inequality (LMI) using the following Lemma 1:

Lemma 1 (S-Procedure): Let a function $f_{m}(\boldsymbol{x}), m \in\{1,2\}$, be defined as

$$
f_{m}(\boldsymbol{x})=\boldsymbol{x}^{H} \boldsymbol{A}_{m} \boldsymbol{x}+2 \Re\left\{\boldsymbol{b}_{m}^{H} \boldsymbol{x}\right\}+c_{m}
$$

where $\boldsymbol{A}_{m} \in \mathbb{H}^{N \times N}, \boldsymbol{b}_{m} \in \mathbb{C}^{N \times 1}$ and $c_{m} \in \mathbb{R}$. The implication $f_{1}(\boldsymbol{x}) \Rightarrow f_{2}(\boldsymbol{x})$ holds if and only if there exists an $\lambda \geq 0$ such that

$$
\lambda\left[\begin{array}{ll}
\boldsymbol{A}_{1}, & \boldsymbol{b}_{1} \\
\boldsymbol{b}_{1}^{H}, & c_{1}
\end{array}\right]-\left[\begin{array}{ll}
\boldsymbol{A}_{2}, & \boldsymbol{b}_{2} \\
\boldsymbol{b}_{2}^{H}, & c_{2}
\end{array}\right] \succeq \mathbf{0} .
$$

To utilize S-procedure, we simply define $f_{1}=\left[\sqrt{\boldsymbol{e}_{I, u}-\boldsymbol{e}_{R, u} \tan \theta} ; \sqrt{\boldsymbol{e}_{R, u}+\boldsymbol{e}_{I, u} \tan \theta}\right]^{T} \boldsymbol{I}_{2 N}$ $\left[\sqrt{\boldsymbol{e}_{I, u}-\boldsymbol{e}_{R, u} \tan \theta} ; \sqrt{\boldsymbol{e}_{R, u}+\boldsymbol{e}_{I, u} \tan \theta}\right] \leq \xi^{2}$, and $f_{2}=\left[\sqrt{\boldsymbol{e}_{I, u}-\boldsymbol{e}_{R, u} \tan \theta} ; \sqrt{\boldsymbol{e}_{R, u}+\boldsymbol{e}_{I, u} \tan \theta}\right]^{T} \operatorname{diag}\left(\boldsymbol{s}_{R} ; \boldsymbol{s}_{I}\right)$ $\left[\sqrt{\boldsymbol{e}_{I, u}-\boldsymbol{e}_{R, u} \tan \theta} ; \sqrt{\boldsymbol{e}_{R, u}+\boldsymbol{e}_{I, u} \tan \theta}\right]+\varrho_{u, 1} \leq 0$. According to S-procedure. The implication $f_{1} \Rightarrow f_{2}$ holds if and only if there exists $\lambda_{u, 1}$ such that the LMI constraint in (13) holds

$$
\begin{aligned}
& {\left[\begin{array}{cc}
\lambda_{u, 1} \boldsymbol{I}_{2 N}-\operatorname{diag}\left(\boldsymbol{s}_{R} ; \boldsymbol{s}_{I}\right), & \mathbf{0} \\
\mathbf{0}, & -\lambda_{u, 1} \xi^{2}-\varrho_{u, 1}
\end{array}\right] \succeq \mathbf{0},} \\
& \text { and } \lambda_{u, 1} \geq 0,
\end{aligned}
$$

As can be seen, a new variable $\xi^{2}$ is introduced to represent the bound of $f_{1}$, which needs to be connected with the known CSI error variance $\sigma_{u}^{2}$ to make the problem solvable. Hence, we further introduce Lemma 2 as follows.

Lemma 2 (links $\xi^{2}$ to CSI error variance $\sigma_{u}^{2}$ ): The value of $\xi^{2}$ can be given as $\xi^{2}=\Phi^{-1}(\delta) \sqrt{N\left(1+\tan ^{2} \theta\right)} \sigma_{u}$ to guarantee the term $\left[\sqrt{\boldsymbol{e}_{I, u}-\boldsymbol{e}_{R, u} \tan \theta} ; \sqrt{\boldsymbol{e}_{R, u}+\boldsymbol{e}_{I, u} \tan \theta}\right]^{T} \boldsymbol{I}_{2 N}$ $\left[\sqrt{\boldsymbol{e}_{I, u}-\boldsymbol{e}_{R, u} \tan \theta} ; \sqrt{\boldsymbol{e}_{R, u}+\boldsymbol{e}_{I, u} \tan \theta}\right] \leq \xi^{2}$ satisfied with probability $\delta$, where $\delta$ can be set to close to 1 , i.e. $\delta=0.99$ [25]. Proof: Please see Appendix A.

Now with the value of $\xi^{2}$, the first constraint in (9) containing infinite possibilities of CSI uncertainties is transformed into a deterministic LMI constraint. Now we handle the second constraint in (9), which can be written as 


$$
\left[\begin{array}{cc}
\lambda_{u, 2} \boldsymbol{I}_{2 N}-\operatorname{diag}\left(\boldsymbol{s}_{R} ; \boldsymbol{s}_{I}\right), & \mathbf{0} \\
\mathbf{0}, & -\lambda_{u, 2} \xi^{2}-\varrho_{u, 2}
\end{array}\right] \succeq \mathbf{0},
$$

and $\lambda_{u, 2} \geq 0$

where $\varrho_{u, 2}=\sigma_{n} \sqrt{\overline{\Gamma_{u}}} \tan \theta-\boldsymbol{h}_{I, u} \boldsymbol{s}_{R}-\hat{\boldsymbol{h}_{R, u}} \boldsymbol{s}_{I}-\hat{\boldsymbol{h}_{R, u}} \boldsymbol{s}_{R} \tan \theta+$ $\hat{\boldsymbol{h}_{I, u}} \boldsymbol{s}_{I} \tan \theta$, and $\xi^{2}$ can be similarly calculated by Lemma 2 . Now the constraint $(C 3)$ containing infinite possibilities is transformed into two deterministic LMI inequalities in (13) and (14), respectively. Now we handle the $k$-th Eve's constraint in $(C 4)$, which can be re-written as

$$
\left\{\begin{array}{l}
\max _{\boldsymbol{e}_{\boldsymbol{k}} \in \boldsymbol{\Delta}} \\
{\left[\boldsymbol{e}_{I, k}+\boldsymbol{e}_{R, k} \tan \theta ; \boldsymbol{e}_{R, k}-\boldsymbol{e}_{I, k} \tan \theta\right]^{T}\left[\boldsymbol{s}_{R} ; \boldsymbol{s}_{I}\right]+\varrho_{k, 1} \leq 0,} \\
\max _{\boldsymbol{e}_{\boldsymbol{k}} \in \boldsymbol{\Delta}} \\
{\left[-\boldsymbol{e}_{I, k}+\boldsymbol{e}_{R, k} \tan \theta ;-\boldsymbol{e}_{R, k}-\boldsymbol{e}_{I, k} \tan \theta\right]^{T}\left[\boldsymbol{s}_{R} ; \boldsymbol{s}_{I}\right]+\varrho_{k, 2} \leq 0}
\end{array}\right.
$$

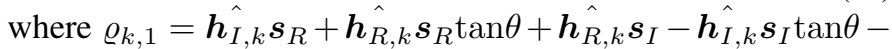
$\sigma_{n} \sqrt{\overline{\Gamma_{k}}} \tan \theta$, and $\varrho_{k, 2}=-\hat{\boldsymbol{h}_{I, k}} \boldsymbol{s}_{R}+\hat{\boldsymbol{h}_{R, k}} \boldsymbol{s}_{R} \tan \theta-\hat{\boldsymbol{h}_{R, k}} \boldsymbol{s}_{I}-$ $\hat{\boldsymbol{h}_{I, k}} \boldsymbol{s}_{I} \tan \theta-\sigma_{n} \sqrt{\overline{\Gamma_{k}}} \tan \theta$. Based on S-procedure introduced above, (15) can be transformed into

$$
\left\{\begin{array}{cc}
{\left[\begin{array}{cc}
\lambda_{k, 1} \boldsymbol{I}_{2 N}-\operatorname{diag}\left(\boldsymbol{s}_{R} ; \boldsymbol{s}_{I}\right), & \mathbf{0} \\
\mathbf{0}^{T} & -\lambda_{k, 1} \zeta^{2}-\varrho_{k, 1}
\end{array}\right],} & \mathbf{0} \\
{\left[\begin{array}{cc}
\lambda_{k, 2} \boldsymbol{I}_{2 N}-\operatorname{diag}\left(\boldsymbol{s}_{R} ; \boldsymbol{s}_{I}\right), & -\lambda_{k, 2} \zeta^{2}-\varrho_{k, 2}
\end{array}\right]} \\
\mathbf{0}^{T} &
\end{array}\right.
$$

where $\zeta^{2}=\Phi^{-1}(\delta) \sqrt{N\left(1+\tan ^{2} \theta\right)} \sigma_{k}$ according to Lemma 2 . Now the non-convex constraints $(C 3)$ and $(C 4)$ are replaced by equivalent LMI constraints. Defining $S=\boldsymbol{s} \boldsymbol{s}^{H}$, the problem can be transformed as

$$
\begin{aligned}
& P 2: \underset{\boldsymbol{s}, \boldsymbol{t}}{\operatorname{argmin}} \frac{\operatorname{Tr}(\boldsymbol{S})}{\eta}+\sum_{n=1}^{N}\left(t_{n} p_{o n}+\left(1-t_{n}\right) p_{o f f}\right), \\
& \text { s.t. }(C 1): \operatorname{Tr}\left(\boldsymbol{S} \boldsymbol{F}_{n}\right) \geq 0, \forall n \in N, \\
& \quad(C 2): \operatorname{Tr}\left(\boldsymbol{S} \boldsymbol{F}_{n}\right) \leq t_{n} p_{D A}, \forall n \in N, \\
& \quad(C 3):(13) \text { and }(14),(C 4):(16), \forall k \in K, \\
& \quad(C 5): t_{n}=\{0,1\}, \forall n \in N, \\
& \quad(C 6):\left[\begin{array}{rr}
\boldsymbol{S} & \boldsymbol{s} \\
\boldsymbol{s}^{T} & 1
\end{array}\right] \succeq 0,
\end{aligned}
$$

where $F_{n}=\operatorname{diag}(\underbrace{0 \ldots 0}_{n-1}, 1, \underbrace{0, \ldots 0}_{N-n})$ is an auxiliary diagonal matrix whose elements are zero except the $n$-th element, $\forall k \in K$. Besides, we notice constraint (C6) guarantees that $\boldsymbol{S}$ is a semi-positive matrix, and hence $\operatorname{Tr}\left(\boldsymbol{S} \boldsymbol{F}_{n}\right) \geq 0$ always holds, $\forall n \in N$. As a result, we can drop the constraint (C1) to reduce the complexity. Now the difficulty lies in the binary constraint in (C5), which evidently equals to

$$
\begin{aligned}
& (C 5 a): t_{n}=[0,1], \forall n \in N, \\
& (C 5 b): \sum_{n=1}^{N} t_{n}-\sum_{n=1}^{N} t_{n}^{2} \leq 0 .
\end{aligned}
$$

where $(C 5 a)$ is the relaxed version of the original constraint, and $(C 5 b)$ confines the value of $t_{n}$ close to 0 or 1 . Introducing a penalty factor $\varphi$, typically of large value, and moving $(C 5 b)$ into the objective function, the objective becomes into $\frac{\operatorname{Tr}(\boldsymbol{S})}{\alpha}+$ $\sum_{n=1}^{N}\left(t_{n} p_{o n}+\left(1-t_{n}\right) p_{o f f}\right)+\varphi\left(\sum_{n=1}^{N} t_{n}-\sum_{n=1}^{N} t_{n}^{2}\right)$, which shares the same optimal design policy and result with the original problem [24]. The last difficulty lies in the non-convex term $\varphi\left(\sum_{n=1}^{N} t_{n}-\sum_{n=1}^{N} t_{n}^{2}\right)$ in the objective term. It can be observed that $\sum_{n=1}^{N} t_{n}-\sum_{n=1}^{N} t_{n}^{2}$ is the difference of two convex functions w.r.t the variable $t_{n}$, and thus can be handled by successive convex approximation such that $\sum_{n=1}^{N} t_{n}-$ $\sum_{n=1}^{N} t_{n}^{2} \leq \sum_{n=1}^{N} t_{n}-\sum_{n=1}^{N}\left(t_{n}^{(i)}\right)^{2}-2 \sum_{n=1}^{N} t_{n}^{(i)}\left(t_{n}-t_{n}^{(i)}\right)$, where $t_{n}^{(i)}$ denotes the value of $t_{n}$ at the $i$-th iteration [22]. Therefore, the optimization becomes into

$$
\begin{aligned}
& P 3: \underset{\boldsymbol{s}, \boldsymbol{t}}{\operatorname{argmin}} \frac{\operatorname{Tr}(\boldsymbol{S})}{\eta}+\sum_{n=1}^{N}\left(t_{n} p_{o n}+\left(1-t_{n}\right) p_{o f f}\right) \\
& \quad+\varphi\left(\sum_{n=1}^{N} t_{n}-\sum_{n=1}^{N}\left(t_{n}^{(i)}\right)^{2}-2 \sum_{n=1}^{N} t_{n}^{(i)}\left(t_{n}-t_{n}^{(i)}\right)\right), \\
& \text { s.t. }(C 2),(C 3),(C 4),(C 5 a): t_{n} \in[0,1],(C 6) .
\end{aligned}
$$

Now the deterministic robust optimization is summarized in Algorithm 1. To tighten the approximation, we update the value of $t_{n}^{(i)}$ in each iteration. The successive convex approximation serves as the upper bound of the original problem, which is iteratively minimized and also lower bounded by the SINR and PHY security constraints. Hence, the convergence of the algorithm is confirmed. Finally, the solver for P3 in (19) is summarized as follows.

$\overline{\text { Algorithm } 1 \text { DA deterministic robust optimization algorithm }}$ (DA-det)

Input: SINR requirements $\overline{\Gamma_{u}}$ and $\overline{\Gamma_{k}}$. Estimated channel $\hat{\boldsymbol{h}_{u}}, \hat{\boldsymbol{h}_{k}}, \forall k \in K$. Power consumption related parameters $\eta, p_{\text {on }}, p_{o f f}, p_{D A}$.

Output: Optimal precoding $s$ and DAs activation/deactivation $t$.

1: repeat

2: $\quad$ Solve optimization problem P3 in (19).

3: $\quad$ Update $t_{n}^{(i)}=t_{n}, \forall n \in N$.

4: $\quad i=i+1$.

5: until Convergence

\section{Simulation Results}

Simulated performance is presented in this section. The number of the Eves $K=2$. The LU's SINR requirement is set to $\overline{\Gamma_{d}}=20 \mathrm{~dB}$, while Eves' SINR should be lower than $\overline{\Gamma_{k}}=-10 \mathrm{~dB}$ to address PHY security. Power consumption related parameters are set to $\eta=40 \%, p_{\text {on }}=500 \mathrm{~mW}$, $p_{o f f}=50 \mathrm{~mW}$, and $p_{D A}=1000 \mathrm{~mW}$, respectively. The PL model in [6] is adopted. The central frequency is set to $2 \mathrm{GHz}$ with $1 \mathrm{MHz}$ bandwidth. The AWGN power spectral density is $-174 \mathrm{dBm} / \mathrm{Hz} . \quad N=16 \mathrm{DAs}$ are uniformly fixed across a 


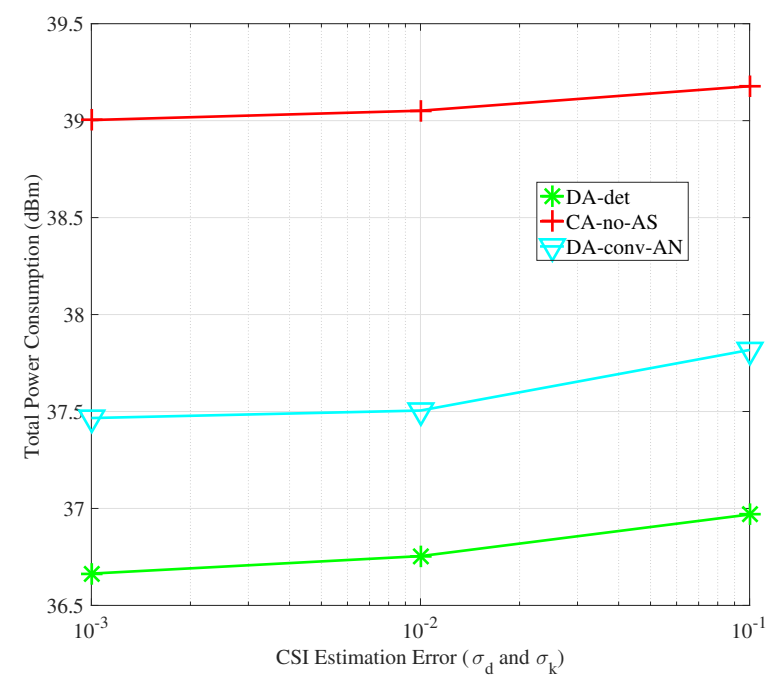

Fig. 2. Impact of the CSI estimation error on the total power consumption.

$100 \times 100 \mathrm{~m}^{2}$ square cell [22]. The LU and Eves are randomly distributed across the map. To highlight how users' positions affect DAs' working modes, the LU and Eves are placed at the coverage edge in Fig. 3, Besides, we select the most related systems as benchmarks. a) CA MISO system without antenna selection (CA-no-AS) [21]. b) DA system with conventional AN (DA-conv-AN) [3].

Fig. 2 shows the CSI estimation error vs. the total power consumption. As can be seen, the proposed algorithm outperforms the two benchmarks, and reason is threefold: a) Benefiting from the antenna selection mechanism, the DAs far from the users may be deactivated for saving power, and thus a usercentric and on-demand network structure is obtained by the proposed algorithm. b) AN is rotated to be constructive even with imperfect CSI, which also endorses less transmission power to achieve a target SINR performance. c) Because of the geographically distributed DAs, the distances between the DAs and users are shortened and the system can always find near DAs to serve the users. With the alleviated PL as well as the lower required transmission power, DA can outperform the CA system in terms of power efficient transmission. Besides, the power consumption of all the algorithms increases with a higher CSI error. Explicitly, the deterministic manner robust optimization needs to keep the positive semi-definite characteristic for the matrices in Eqs. (13), (14) and (16). This mathematically requires that all the leading principal minors in the matrices nonnegative. Hence, with a higher CSI uncertainty, the amplitude of precoder (also the transmission power) needs to be property increased. As a result, the total power consumption increases in a tough CSI estimation scenario.

Fig. 3 shows how antennas' modes (activation or deactivation) are affected by the users' positions. To highlight the usercentric network structure provided by the proposed algorithm, the users in Fig. 3(a) are placed at edge area. As can be seen from Fig. 3(b) that, those DAs close to the users have higher probabilities of working while other DAs far from the users

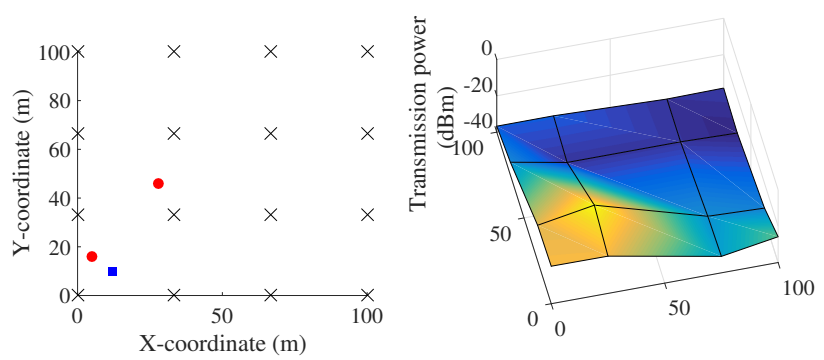

Fig. 3. (a) DAs and users deployment, where black cross indicates the DAs. Red and blue dots indicate the Eves and LU, respectively. (b) Impact of the Eves and LU' positions on antennas' modes in DA systems.

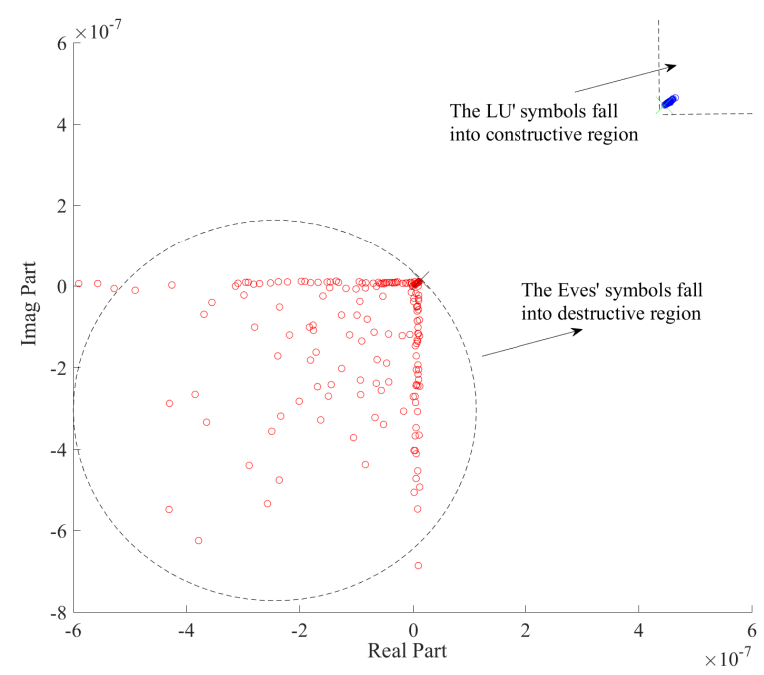

Fig. 4. The probabilities of the received symbols of the LU and Eves falling into constructive and destructive regions, where red dots denote the received symbols of the Eves and blue dots denote the received symbols of the LU.

have higher probabilities of being deactivated to save power. This is because in terms of power efficient design, letting those DAs far from the users transmit signal is not power efficient due to the severe propagation attenuation. As can be expected, since no antenna selection is involved in [21], all antennas are activated ignoring the users' positions.

Fig. 4 shows the probabilities of the received symbols of the LU and Eves falling into different regions. Since infinite possibilities of CSI uncertainties are presented in an imperfect channel estimation scenario, we need to transform constraints (C3) and (C4) into equivalent LMIs. Simulation results verify our analysis that by using S-procedure, the LU' received symbol always fall into constructive region confined by constraint (C3), while the Eves' symbols always fall into destructive region confined by constraint $(\mathrm{C} 4)$. As a result, the robustness of the proposed algorithm is guaranteed.

\section{CONCLUSiOnS}

We have proposed a novel power efficient algorithm for DA systems by jointly optimizing antenna activation/deactivation and precoding, which significantly reduces the total power 
consumption compared to the two benchmarks in [3] and [21]. In the presence of infinite probabilities of CSI uncertainties, the LU's QoS requirement and the PHY security against the Eves can be always guaranteed, which indicates the robustness of the proposed algorithm. Moreover, since DAs can flexibly adjust their working modes thanks to the enhanced degree of freedom, the proposed algorithm also facilitates a more flexible and user-centric network structure over the two benchmarks in [3] and [21]. At last, the performance of the proposed algorithm has been verified by our simulation results.

APPENDIX A

\section{PRoOF OF LEMMA 2}

Since the element of CSI error vector $e_{u}$ follows normal distribution such that $\mathcal{C N}\left(0, \sigma_{u}^{2}\right)$, the element of real part $\boldsymbol{e}_{R, u}$ and imaginary part $\boldsymbol{e}_{I, u}$ follows $\mathcal{C N}\left(0, \frac{\sigma_{u}^{2}}{2}\right)$. Hence, $f_{1}$ can be expanded as $(1+\tan \theta) \boldsymbol{e}_{I, u}+(1-\tan \theta) \boldsymbol{e}_{R, u} \leq \xi^{2}$, which can be approximately seen as the probability distribution function (pdf) of a normal distributed variable such that

$$
\operatorname{Pr}\left\{(1+\tan \theta) \boldsymbol{e}_{I, u}+(1-\tan \theta) \boldsymbol{e}_{R, u} \leq \xi^{2}\right\}=\delta,
$$

where $\delta$ physically represents the probability of Eq. (20) can be satisfied. The value of $\delta$ can be set to close to 1 , i.e. $\delta=$ 0.99 , meaning (20) is satisfied with high probability. Since we know the term $(1+\tan \theta) \boldsymbol{e}_{I, u}+(1-\tan \theta) \boldsymbol{e}_{R, u}$ in Eq. (20) follows $\mathcal{C N}\left(0, N \sigma_{u}^{2}\left(1+\tan \theta^{2}\right)\right)$. By normalizing it into a stand normal distributed variable, (20) can be written as a cumulative distribution function (cdf) as $\Phi\left\{\frac{\xi^{2}}{\sqrt{N\left(1+\tan ^{2} \theta\right)}}\right\}=\delta$, where $\Phi(\cdot)$ is the cdf of a standard normal distributed variable. Defining $\Phi^{-1}(\cdot)$ as the inverse function of $\Phi(\cdot)$, finally we get

$$
\xi^{2}=\Phi^{-1}(\delta) \sigma_{u} \sqrt{N\left(1+\tan ^{2} \theta\right)}
$$

and the value $\zeta^{2}$ (linked to the Eves' CSI error variance $\sigma_{k}$ ) can be calculated similarly.

\section{REFERENCES}

[1] H. Lei, Z. Dai, K. Park, W. Lei, G. Pan, and M. S. Alouini, "Secrecy outage analysis of mixed RF-FSO downlink SWIPT systems," to appear in IEEE Trans. Commun., vol. -, no. -, pp. 1-1, Dec. 2018, DOI: 10.1109/TCOMM.2018.2865944.

[2] K. Cumanan, Z. Ding, B. Sharif, C. Tian, and K. Leung, "Secrecy rate optimization for a MIMO secrecy channel with a multiple-antenna eavesdropper," IEEE Trans. Veh. Technol., vol. 63, no. 4, pp. 1678-1690, May 2014.

[3] D. W. K. Ng and R. Schober, "Secure and green SWIPT in distributed antenna networks with limited backhaul capacity," IEEE Trans. Wireless Commun., vol. 14, no. 9, pp. 5082-5096, Dec. 2015.

[4] X. Gao, L. Dai, S. Han, C-L. I, and R. W. Heath, "Energy efficient hybrid analog and digital precoding for mmwave MIMO systems with large antenna arrays," IEEE J. Sel. Areas Commun., vol. 34, no. 4, pp. 998-1009, Mar. 2016.

[5] Z. Wei, X. Zhu, S. Sun, and Y. Huang "Energy efficiency oriented cross layer resource allocation for multiuser full-duplex decode-and-forward indoor relay systems at $60 \mathrm{GHz}$," IEEE J. Sel. Areas Commun., vol. 34, no. 12, pp. 3366-3379, Dec. 2016.

[6] J. Pardo, J. Rodrguez, and L. Llcer, "Polarized Indoor MIMO channel measurements at $2.45 \mathrm{GHz}$," IEEE Trans. Antenna Propagat., vol. 56, no. 12, pp. 3818-3828, Dec. 2006.

[7] J. Wang and L. Dai, "Downlink rate analysis for virtual-cell based largescale distributed antenna systems," IEEE Trans. Wireless Commun., vol. 15, no. 3, pp. 1998-2011, Mar. 2016.
[8] J. Joung, Y. K. Chia, and S. Sun, "Energy-efficient, large-scale distributed antenna system (L-DAS) for multiple users," IEEE Trans. Signal Process., vol. 8, no. 5, pp. 964-965, Oct. 2014.

[9] S. Goel and R. Negi, "Guaranteeing secrecy using artificial noise," IEEE Trans. Wireless Commun., vol. 7, no. 4, 2180-2189, Jun. 2008.

[10] Q. Li and W. K. Ma, "Spatially selective artificial noise aided transmit optimization for MISO multi-eves secrecy rate maximization," IEEE Trans. Signal Process., vol. 61, no. 10, pp. 2704-2717, May 2013.

[11] H. Wang, C. Wang, D. W. K. Ng, M. H. Lee, and J. Xiao, "Artificial noise assisted secure transmission for distributed antenna systems," IEEE Trans. Signal Process., vol. 64, no. 15, pp. 4050-4064, Aug. 2016.

[12] K. Gao, Y. Gao, and G. Ascheid, "Security constraint power allocation in MU-massive-MIMO with distributed antennas," IEEE Trans. Wireless Commun., vol. 15, no. 12, pp. 8139-8153, Dec. 2016.

[13] C. Masouros and E. Alsusa, "A novel transmitter-based selective precoding technique for DS/CDMA systems," IEEE Sig. Process. Lett., vol. 14, no. 9, pp. 637-640, Sep. 2007.

[14] E. Alsusa and C. Masouros, "Adaptive code allocation for interference management on the downlink of DS-CDMA systems," IEEE Trans. Wireless Commun., vol. 7, no. 7, pp. 2420-2424, Jul. 2008.

[15] C. Masouros, E. Alsusa, "Two-stage transmitter precoding based on data-driven code-hopping and partial zero forcing beamforming for MCCDMA communications, IEEE Trans. Wireless Commun., vol. 8, no. 7, pp. 3634-3645, Jul. 2009.

[16] P. V. Amadori and C. Masouros, "Large scale antenna selection and precoding for interference exploitation," IEEE Trans. Commun., vol. 65, no. 10, pp. 4529-4542, Oct. 2017.

[17] K. L. Law, C. Masouros, and M. Pesavento, "Transmit precoding for interference exploitation in the underlay cognitive radio Z-channel," IEEE Trans. Signal Process., vol. 65, no. 14, pp. 3617-3630, Jul. 2017.

[18] FA Khan, C Masouros, and T Ratnarajah, "Interference-driven linear precoding in multiuser MISO downlink cognitive radio network," IEEE Trans. Veh. Technol., vol. 61, no. 6, pp. 2531-2543, Jul. 2012.

[19] P. V. Amadori and C. Masouros, "Constant envelope precoding by interference exploitation in phase shift keying modulated multiuser transmission," IEEE Trans. Wireless Commun., vol. 16, no. 1, pp. 538-550, Jan. 2017.

[20] F. Liu, C. Masouros, P. Amadori, and H Sun, "An efficient manifold algorithm for constructive interference based constant envelope precoding," IEEE Signal Process. Lett.,, vol. 24, no. 10, pp. 1542-1546, Jul. 2017.

[21] M R. A. Khandaker, K. Masouros, and K. K. Wong, "Constructive interference based secure precoding: a new dimension in physical layer security," IEEE Trans. Inf. Foren. Sec., vol. 13, no. 9, pp. 2256-2268, Sep. 2018.

[22] Z. Wei, S. Sun, X. Zhu, Y. Huang, and J. Wang, "Energy-efficient hybrid duplexing strategy for bidirectional distributed antenna systems," IEEE Trans. Veh. Technol., vol. 67, no. 6, pp. 5096-5110, Apr. 2018.

[23] S. Boyd and L. Vandenberghe, Convex Optimization. Cambridge, U.K.: Cambridge Univ. Press, 2004.

[24] D. W. K. Ng, Y. Wu, and R. Schober, "Power efficient resource allocation for full-duplex radio distributed antenna networks," IEEE Trans. Wireless Commun., vol. 15, no. 4, pp. 2896-2911, Apr. 2016.

[25] Z. Wei, C. Masouros, K. Wong, and X. Kang, "Multi-cell interference exploitation: A new dimension in cell coordination," arXiv:1901.04058, Jan. 2019 